\title{
MÉTODOS DE DETECÇÃO DE CÁRIE: DO TRADICIONAL ÀS NOVAS TECNOLOGIAS DE EMPREGO CLÍNICO
}

\section{METHODS FOR DETECTION OF DENTAL CARIES: FROM TRADITIONAL TO NEW TECHNOLOGIES FOR CLINICAL USE.}

\author{
Jorge César Borges Leão Filho* \\ Thayse Rodrigues de Souza**
}

\begin{abstract}
RESUMO
O desenvolvimento de novos métodos de detecção de cárie tem o objetivo de facilitar o diagnóstico de lesões incipientes de cárie, suprindo a deficiência dos métodos convencionais. Pretende-se avaliar os métodos de detecção de cárie citados na literatura e compará-los segundo sua especificidade e sensibilidade, desde os mais tradicionais até os mais recentes. Durante o século 20, critérios tátil-visuais foram usados para o diagnóstico de cárie dental; estes detectavam apenas a presença de cavitação. Com compreensão crescente do processo da cárie dental, a criação de sistemas diagnósticos sensíveis e específicos é necessária para permitir a detecção precoce de desmineralização. Para tanto, o avanço da tecnologia tem auxiliado na criação de novos métodos para a detecção de cárie. Esses novos métodos são baseados na mensuração da diferença de condutividade elétrica entre tecido sadio e tecido lesado, em imagens obtidas através de radiação X e na dispersão da luz visível nos tecidos dentais.
\end{abstract}

DESCRITORES: Cárie dentária • Diagnóstico

\section{ABSTRACT}

The development of new methods of caries detection aims to facilitate the diagnosis of incipient caries lesions, supplying the deficiency of conventional methods. The purpose of this review is to evaluate methods of detecting caries in literature and compare them according to their specificity and sensitivity, from the most traditional to the most recent. During the 20th century, visual-tactile criteria were used for the diagnosis of dental caries; they detected only the presence of cavitation. With growing understanding of the process of dental caries, the creation of sensitive and specific diagnostic systems is needed to allow early detection of demineralization. Thus, the advancement of technology has helped in creating new methods for detection of caries. These new methods are based on the measurement of electrical conductivity difference between healthy tissue and injured tissue, in images obtained by X-rays and visible light scattering in dental tissues.

DESCRIPTORS: Dental caries • Diagnosis 
LEÃO FILHO JCB

SOUZA TR

MÉTODOS DE

DETECÇÃO DE

CÁRIE: DO

TRADICIONAL

ÀS NOVAS

TECNOLOGIAS DE

EMPREGO CLÍNICO

\section{$254 \ldots$}

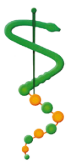

REV, ODONTOL.

UNIV, CID, SÃO PAULO

2011; 23(3): 25365, SET-DEZ

\section{N T RO DUÇÃO}

A cárie é considerada hoje como resultante do desequilíbrio nos processos de desmineralização e remineralização (Fejerskov e Kidd $\left.{ }^{1}, 2005\right)$. No presente momento, devido ao maior contato da população com compostos fluoretados, notam-se alterações no padrão de desenvolvimento das lesões de cárie, tais como a sua progressão mais lenta e a cavitação mais tardia. Com esse novo padrão de cárie, a detecção e a avaliação da extensão das lesões, principalmente em seus estágios iniciais de desenvolvimento, tornaram-se uma tarefa complicada (Mialhe et al. $^{2}, 2005$, Ismail $\left.{ }^{3}, 1997\right)$.

A atenção é dada hoje a pesquisas científicas e ao cuidado em detectar a doença cárie avaliando o estágio das lesões (Nyvad $^{4}$, 2004). No entanto encontra-se ainda dificuldade em detectar lesões de cárie iniciais através dos métodos convencionais, e as novas tecnologias têm surgido no intuito de suprir essas deficiências (Murdoch-Kinch e McLean ${ }^{5}$, 2003).

Durante muito tempo os principais métodos utilizados na clínica para detecção e avaliação da extensão de lesões de cárie por cirurgiões-dentistas foram exame clínico visual, tátil e radiográfico, classificados como métodos tradicionais ou convencionais de detecção. Outros métodos, chamados de complementares, são descritos na literatura: transiluminação por fibra óptica (FOTI), o uso de corantes, a separação interdental e os refinamentos nos métodos tradicionais, tais como a radiografia digitalizada, lupas, espelho dental com luz e a câmera intraoral. Atualmente, novas tecnologias e métodos têm sido elaborados e validados com o objetivo de suprir as falhas de outros métodos, e nesse contexto se enquadram o Laser Fluorescente de baixa frequência (aparelho DIAGNOdent - Kavo,Alemanha), a medição da resistência elétrica oferecida pelo elemento dental (aparelho ECM - LODE, Holanda), o aparelho DIFOTI, o Quantitative Light-Induced Fluorescence (QLF) e a Tomografia computadorizada (Mialhe et al. ${ }^{2}, 2005$, Murdoch-Kinch e McLean ${ }^{5}$, 2003).

$$
\text { REVISÃO DA LITERATURA }
$$

Um método de detecção ideal deve ser confiável, capaz de detectar lesões de cárie em estágio inicial, diferenciar lesões reversíveis das irreversíveis e permitir sua documentação. Além disso, deve ter custo acessível, promover conforto para o paciente, rapidez e facilidade de execução, aplicabilidade a todos os sítios dos dentes com a mesma eficiência, características estas essenciais para um método diagnóstico ser considerado adequado (Marinho e Pereira $^{6}$, 1998). Um método de detecção de cáries deveria oferecer, dentre outras características, uma alta sensibilidade e alta especificidade (Lussi e Francescut ${ }^{7}$, 2003).

Sensibilidade e especificidade são conceitos utilizados para comparar a eficiência de diferentes métodos de detecção de cárie. A sensibilidade mede a capacidade do teste em identificar corretamente a doença entre aqueles que a possuem; já a especificidade mede a capacidade do teste em excluir corretamente aqueles que não possuem a doença. Ambos são medidos em escalas de 0 a 1 , quanto mais próximos os valores de 1 mais específico ou sensível o método avaliado (Fejerskov e Kidd $\left.{ }^{1}, 2005\right)$.

\section{Inspeção Visual}

O método de inspeção visual para a detecção de lesões de cárie baseia-se na busca por alterações físicas no elemento dental, tais como cavitação, presença de sombreamento, além de mudanças na translucidez e na textura do esmalte. A observação da presença ou não de agente etiológico da cárie (biofilme dental) também é feita durante a inspeção visual (Fejerskov e Kidd ${ }^{1}$, 2005).

Para a realização da inspeção visual, as superfícies dentárias a serem examinadas devem estar devidamente limpas, tendo sido removido o biofilme, restos de alimento ou qualquer outro fator que impeça a visualização da superfície. Porém, antes da remoção do biofilme, é sensato observar a sua localização, pois a presença de depósitos bacterianos é relevante para se avaliar a atividade de uma lesão (Fejerskov e Kidd ${ }^{1}, 2005$, Cortes et al. $\left.{ }^{8}, 2003\right)$.

Uma mancha branca é a manifestação primária de uma lesão de cárie, e ela pode ser observada clinicamente, sem a seca- 
gem da superfície do dente, após duas semanas de desenvolvimento do biofilme dental sem interferências externas. Porém, mediante uma secagem prévia da superfície do esmalte, a mancha branca pode ser visualizada após uma semana de desenvolvimento sem interferências externas do biofilme dental. Quando a lesão é visualizada com uma superfície úmida, provavelmente ela já atravessou toda a extensão do esmalte e já houve desmineralização da dentina; porém quando a mancha branca é vista apenas mediante a secagem, ela provavelmente se encontra superficialmente no esmalte (Fejerskov e Kidd $^{1}$, 2005, Kidd e Fejerskov ${ }^{9}$, 2004).

A inspeção visual é um método de detecção de cárie que apresenta uma alta sensitividade e uma baixa especificidade para lesões de esmalte, e uma alta especificidade e uma baixa especificidade para lesões cariosas em dentina. Os dados numéricos estão expressos na Tabela II (Kidd e Fejerskov ${ }^{9}$, 2004).

Uma das maiores dificuldades nesse tipo de método é diferenciação entre lesões de esmalte profundas e lesões de esmalte com envolvimento da dentina superficial (Kidd e Fejerskov ${ }^{9}, 2004$ ).

Para o exame visual, o valor de kappa para a concordância inter-observadores varia entre 0,61 e 0,79, e isso demonstra que há uma substancial concordância entre diferentes examinadores que usaram a mesma técnica (Hintze et al. ${ }^{10}, 1998$ ).

Atualmente tem-se dado mais ênfase para a aparência clínica da lesão quando é necessário tomar uma decisão sobre a forma de tratamento a ser aplicada (se invasivo ou não); dessa forma, a profundidade da lesão em seu aspecto radiográfico não deve ser um parâmetro absoluto para escolher a forma de tratamento adequado. É válido lembrar que a decisão por um método invasivo é baseada na suposição de que a extensão da desmineralização perante um complexo de sintomas não pode ser estabilizada por meios preventivos (Hintze et al. $\left.{ }^{10}, 1998\right)$.

Para auxiliar a inspeção visual em lesões nas faces interproximais, pode ser utilizado o método de separação dental com anéis de borracha. O anel de borracha é mantido entre um dente e seu vizinho du- rante dois dias, permitindo que o espaço interdental seja aumentado, facilitando assim a inspeção visual e o procedimento restaurador (caso necessário) (Hintze et al. $\left.{ }^{10}, 1998\right)$. Para a detecção de lesões cariosas proximais a inspeção visual possui uma sensibilidade entre 0,58 e 0,74 e especificidade entre 0,83 e 0,97 (Eggertsson et al. $\left.{ }^{11}, 1999\right)$.

Em um estudo de Hintze et al. ${ }^{10}$ (1998), após a avaliação pelo método de inspeção visual simples de 390 faces proximais, dois terços foram consideradas sadias; porém, depois da separação dentária em algumas dessas superfícies foram encontradas cavitações $(0,5-2,6 \%)$. Esses achados diferem dos de um estudo brasileiro (De Araujo et al.12, 1992), onde, após a separação dentária, em $21 \%$ das faces consideradas sadias após inspeção visual simples encontraram-se cavitações.

A técnica de separação não pode ser considerada como um padrão ouro para a validação de outros métodos de detecção de cárie, porém constitui um método auxiliar valioso para a inspeção visual convencional e no exame radiográfico, principalmente quando é preciso avaliar a necessidade de um tratamento invasivo (Hintze et al. ${ }^{10}$, 1998).

\section{Inspeção Tátil}

Ao longo de muitos anos, os dentistas, durante sua formação, eram ensinados a "atacar", com um explorador de ponta fina, as regiões suspeitas de lesões de cárie. Tal procedimento era realizado com o intuito de fazer um teste, no qual era percebida a presença ou não de alguma resistência durante a retirada da sonda após uma exploração firme. Se a sonda prendesse, isso seria indicativo de lesão de cárie (Fejerskov e Kidd $\left.{ }^{1}, 2005\right)$.

A sondagem com o explorador é um dos mais antigos métodos de detecção de cárie, e possui vantagens como baixo custo, alta especificidade e fácil execução. Porém, suas desvantagens são mais relevantes, e por isso esse método é considerado antiquado e vem tendo seu uso reduzido entre os dentistas (Haak et al. ${ }^{13}, 2002$, Kuhnisch et al. $\left.{ }^{14}, 2007\right)$. Essas desvantagens incluem sua baixa sensibilidade, a possibilidade de transferência
LEÃO FILHO JCB

SOUZA TR

MÉTODOS DE DETECÇÃO DE

CÁRIE: DO

TRADICIONAL

ÀS NOVAS

teCNOLOGIAS DE

EMPREGO CLÍNICO

$\therefore 255$

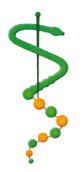

REV, ODONTOL.

UNIV, CID. São

PAULO

2011; 23(3): 253-

65, SET-DEZ 
LEÃO FILHO JCB

SOUZA TR

MÉTODOS DE

DETECÇÃO DE

CÁRIE: DO

TRADICIONAL

ÀS NOVAS

TECNOLOGIAS DE

EMPREGO CLÍNICO

\section{6 。}

REV, ODONTOL.

UNIV. CID, SÃO

PAULO

$2011 ; 23(3): 253-$

65, SET-DEZ de microorganismos cariogênicos de um sítio para outro e, por fim, a mais importante de todas, a possibilidade de quebrar a integridade da superfície de uma lesão incipiente de esmalte, transformando uma lesão subsuperficial passível de remineralização em uma lesão cavitada, e além disso acelerando o desenvolvimento da lesão cariosa (Fejerskov e Kidd', 2005, Lussi e Francescut ${ }^{7}, 2003$, Haak et al. ${ }^{13}$, 2002, Kuhnisch et al. ${ }^{14}$, 2007).

Ao microscópio eletrônico, as marcas da sondagem com um explorador de ponta ativa fina no esmalte são observadas como depressões superficiais, que podem ser vistas como impressões lineares (arranhões) nas vertentes de cúspides assim com no fundo de fissuras de acordo com a espessura da ponta ativa do explorador. A quebra de porções de esmalte também é visível ao microscópio eletrônico, deixando expostos os prismas que formam a estrutura do esmalte. Os danos causados pela exploração variam entre $100 \mu \mathrm{m}$ e $2 \mathrm{~mm}$ de diâmetro (Kuhnisch et al. ${ }^{14}$, 2007).

A inspeção tátil possui uma reprodutibilidade intraexaminadores de ruim para moderada (valor de kappa variando entre $0,24$ e 0,66$)$; já a reprodutibilidade interexaminadores é péssima, possuindo valores de kappa que oscilam entre -0,08 e -0,26 (Ekstrand et al. $\left.{ }^{15}, 2005\right)$ (Tabela I).

A sondagem com o uso de um explorador não aumenta a precisão do exame clínico Fejerskov e Kidd , (2005); por isso a inspeção visual de uma superfície dental limpa e seca deveria ser o método de primeira escolha para a detecção rotineira de cáries. Outros métodos podem ser utilizados como auxiliares da inspeção visual satisfatoriamente, são eles a radiografia interproximal e a fluorescência a laser (Kuhnisch et al. ${ }^{14}$, 2007).

\section{Monitor elétrico de cáries (ECM)}

O ECM é um sistema de detecção de cáries oclusais baseado na observação de uma alteração no comportamento de uma corrente elétrica ao atravessar a lesão cariosa (Fejerskov e Kidd ${ }^{1}$, 2005, Kuhnisch et al. $\left.{ }^{16}, 2006\right)$.

Com o processo de desmineralização do tecido dentário há uma mudança na condutância elétrica do dente, até mesmo quando a superfície permanece aparentemente intacta. Durante a desmineralização do esmalte são criados microporos subsuperficiais em sua estrutura (Hintze et al. $\left.{ }^{10}, 1998\right)$ que são preenchidos por saliva (George e Stookey ${ }^{17}$ ). A existência de saliva nas porosidades subsuperficiais do esmalte é responsável pelo aumento da condutância elétrica nas lesões de esmalte, e a diferença de condutividade entre tecido sadio e tecido lesado é a base para o uso do ECM (Tam e McComb $\left.{ }^{18}, 2001\right)$.

Esse método possui alta sensibilidade e alta especificidade, possuindo geralmente uma sensibilidade superior à inspeção visual e aos exames radiográficos, porém possuindo uma especificidade menor. Os valores de sensibilidade e especificidade estão expressos na Tabela II. Em um estudo in vivo a performance no diagnóstico foi superior ao método radiográfico Bite-wing (Kuhnisch et al. ${ }^{16}, 2006$, Tam e McComb ${ }^{18}$, 2001).

O monitor elétrico de cáries é um aparelho utilizado quase exclusivamente por grupos de pesquisa, não sendo utilizado rotineiramente nos consultórios odontológicos por consumir muito tempo em seu protocolo de utilização e também devido ao seu alto custo (Kuhnisch et al. ${ }^{16}, 2006$ ).

Para a utilização do aparelho existem dois métodos. No método de aplicação local-específico a sonda (eletrodo de medição) é colocada na fissura e então a condutância daquele local é medida. Antes do posicionamento da sonda a superfície oclusal deve ser seca com jato de ar, para que a corrente não "escape" através da camada de saliva que envolve todo o dente e os tecidos moles. Esse método tem algumas desvantagens. A primeira é que a área de mensuração da condutância fica limitada à região que circunda a sonda, e assim apenas uma pequena área do dente pode ser avaliada por vez (Fejerskov e Kidd $^{1}$, 2005). Outra desvantagem é que, após a secagem, o examinador tem pouco tempo para fazer a mensuração, isso porque a saliva voltará à superfície oclusal rapidamente (Kuhnisch et al. ${ }^{16}, 2006$ ). Como alternativa a essa técnica, a superfície oclusal pode ter sua condutância medida como um todo cobrindo-a com um 
meio contendo eletrólitos no qual o eletrodo é inserido (Fejerskov e Kidd' ${ }^{1}, 2005$ ).

Durante a sondagem com o eletrodo a superfície oclusal pode ser danificada, especialmente quando repetidas mensurações forem realizadas. Para haver alguma melhora nesse aspecto, a configuração da ponta exploradora (eletrodo) deveria ser mudada, com a intenção de limitar os danos causados em lesões superficiais fragilizadas (Kuhnisch et al. $\left.{ }^{16}, 2006\right)$.

\section{Tomografia}

4.1 Optical Coherence Tomograhy (OCT)

O termo Tomografia primeiramente foi usado para descrever técnicas radiográficas por secção. A imagem derivada de uma tomografia representa uma "fatia" selecionada de determinada estrutura, e isso é uma grande vantagem, porque evita a superposição de estruturas que pode ser observada em imagens radiográficas (Otis et al. $\left.{ }^{19}, 2000\right)$.

O OCT cria imagens bidimensionais dos tecidos, de modo que as estruturas são vistas através de cortes, sendo os detalhes gerados pela diferenciação entre os fótons dispersos, transmitidos ou refletidos. Esse método ainda está em fase de aperfeiçoamento e pesquisa para ser utilizado em clínicas odontológicas (Otis et al. ${ }^{19}, 2000$ ).

As imagens obtidas através do OCT remontam detalhes microestruturais dos tecidos moles e periodontais, identificando possíveis doenças periodontais ou perdas de osso alveolar. Esse método é potencialmente o mais sensível para detectar cáries recorrentes e para avaliar a adaptação marginal de restaurações (Otis et al. ${ }^{19}$, 2000).

\subsection{Tomografia computadorizada local cone beam (LCT)}

A tomografia computadorizada torna mais fácil a detecção de cáries secundárias, através de suas imagens bidimensionais em "fatia", porém não mostra nenhuma melhoria significativa na detecção de cárie proximal (Kalathingal et al. ${ }^{20}, 2007$ ).

O desenvolvimento da tomografia computadorizada cone beam tem sido revolucionária, pois possibilita a obtenção de imagens tridimensionais detalhadas sem expor o paciente a altas doses, o que faz com que esse tipo de tecnologia ganhe cada vez mais espaço na Odontologia (Kalathingal et al.20, 2007).

Quando testada a eficácia em detectar cáries proximais, os resultados obtidos através do LCT foram superiores àqueles conseguidos com a utilização de radiografias interproximais, porém a diferença não foi muito grande (Kalathingal et al. ${ }^{20}$, 2007).

A diferença na sensibilidade entre os dois métodos é significante, porém a diferença no valor da especificidade não é. A comparação dos valores de especificidade e sensibilidade entre o LCT e a radiografia bite-wing é demonstrada na Tabela III (Kalathingal et al. $\left.{ }^{20}, 2007\right)$.

\section{Métodos de detecção de cáries} baseados na luz visível

Os cristais de hidroxiapatita que formam grande parte da estrutura do esmalte se encontram muito densamente unidos, o que dá a esse tecido dental um aspecto de vidro translúcido. A cor amarelado-esbranquiçada característica dos dentes é o resultado do efeito óptico causado pela dentina que se encontra imediatamente abaixo do esmalte (Fejerskov e Kidd ${ }^{1}$, 2005).

Quando a luz é lançada sobre o dente ela pode se difundir, ou ser absorvida no seu interior. Na lesão de mancha branca o processo de difusão ocorre mais intensamente que no esmalte sadio, fazendo com que a lesão apareça com um tom de branco mais intenso, pois a luz é refletida antes de alcançar a dentina (Fejerskov e Kidd $\left.^{1}, 2005\right)$.

\subsection{Transiluminação por Fibra Óptica (FOTI)}

O FOTI é um método de detecção qualitativo de cáries que se baseia na transiluminação dos dentes, a partir da qual a observação de sombras na estrutura dental é indicativa de lesão cariosa (Fejerskov e Kidd $\left.^{1}, 2005\right)$. Esse método tem sido muito utilizado para a detecção de cáries interproximais, assim como para a avaliação da profundidade de lesões oclusais (Cortes et al. $\left.{ }^{8}, 2003\right)$.

A produção desse sombreamento em
LEÃO FILHO JCB

SOUZA TR

MÉTODOS DE DETECÇÃO DE CÁRIE: DO

TRADICIONAL

ÀS NOVAS

TECNOLOGIAS DE

EMPREGO CLÍNICO
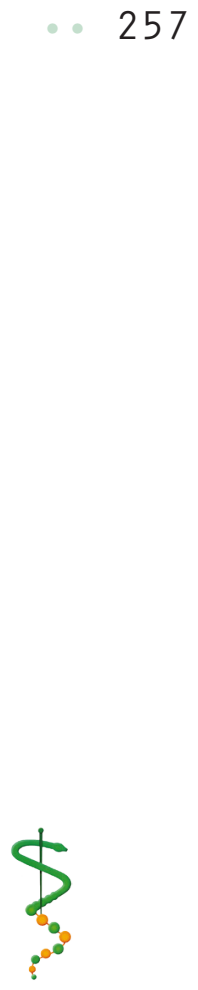

REV, ODONTOL.

UNIV. CID. SÃo

PAULO

2011; 23(3): 253 -

65, SET-DEZ 
LEÃO FILHO JCB

SOUZA TR

MÉTODOS DE

DETECÇÃO DE

CÁRIE: DO

TRADICIONAL

ÀS NOVAS

TECNOLOGIAS DE

EMPREGO CLÍNICO

$258 \ldots$

REV. ODONTOL,

UNIV, CID, SÃO

PAULO

$2011 ; 23(3): 253-$

65, SET-DEZ
I SSN 1983-5183

lesões de esmalte é baseada na existência de porosidades na estrutura desse esmalte, e que, por esse motivo, a estrutura dentária afetada tem um índice de transmissão de luz mais baixo que o do esmalte sadio, produzindo um sombreamento de aspecto acinzentado e opaco. Nas lesões em dentina, o sombreamento assume uma coloração marrom-alanranjada (Cortes et al. ${ }^{8}$, 2003, George e Stookey ${ }^{17}$ ).

O aparelho FOTI é projetado para fornecer um feixe luminoso intenso, transmitido através de um cabo de fibra ótica até uma ponta especialmente projetada para usar a transiluminação nas superfícies proximais dos dentes posteriores. Esse método pode ser usado em faces oclusais tão bem quanto nas superfícies proximais dos dentes (George e Stookey ${ }^{17}$ ).

O desempenho do FOTI para a detecção de cáries de dentina imediatamente abaixo da junção amelo-dentinária é superior àquele obtido com o uso da técnica de radiografia interproximal. Esse método possui alta sensibilidade e baixa especificidade para lesões em esmalte; já para lesões em dentina a transiluminação por fibra óptica apresenta alta especificidade e baixa sensibilidade (Eggertsson et al. ${ }^{11}$, 1999). Os valores de especificidade e sensibilidade para o FOTI estão representados na Tabela II.

Quando comparado com outros métodos (DIAGNOdent e combinação FOTI/ inspeção visual), o FOTI apresentou um escore de correlação com o exame histológico intermediário $(0,64)$, enquanto que o DIAGNOdent e a combinação FOTI/ inspeção visual obtiveram escores de 0,42 e 0,66 respectivamente. Além disso, o FOTI apresenta um valor de kappa para a reprodutibilidade intra-examinador de 0,78 , considerado alto (Cortes et al. ${ }^{8}$, 2003).

O FOTI tem-se mostrado mais eficaz para a detecção de cavitação e para a mensuração da profundidade em lesões interproximais do que os métodos convencionais de exame clínico (Ricketts et al. $\left.{ }^{21}, 2007\right)$. Esse método possui, ainda, um protocolo simples, sendo não invasivo e confortável para o paciente, porém possui a limitação de não detectar lesões de cárie secundária (Marinho e Pereira6,
1998).

Na tentativa de melhorar o desempenho do FOTI, foi criado outro aparelho, chamado DIFOTI. Esse novo aparelho é um avanço tecnológico, pelo qual as imagens são capturadas com uma câmera digital acoplada à peça de mão (CCD) e emitidas a um computador para a análise $\left(\right.$ George e Stookey ${ }^{17}$ ).

O FOTI é uma alternativa às radiografias interproximais e maiores desenvolvimentos e pesquisas podem permitir que a técnica se torne um método de diagnóstico de rotina na clínica odontológica (Marinho e Pereira ${ }^{6}$, 1998).

\subsection{Quantitative light-induced Fluo- rescence (QLF)}

A desmineralização do tecido dentário resulta na perda de sua fluorescência natural e é nessa propriedade do tecido dental que se baseia o QLF. Nesse método de detecção a diminuição da fluorescência é indicativa da perda de minerais das estruturas dentais (Fejerskov e Kidd'1, 2005).

O equipamento QLF possui uma fonte luminosa acoplada à peça de mão, que deve ser direcionada para a região a ser examinada. As imagens fluorescentes são capturadas usando-se uma câmera digital também acoplada à peça de mão; então os dados são coletados, armazenados, e analisados por software de computador. A imagem, por fim, é exibida em um monitor (George e Stookey ${ }^{17}$ ).

Os valores de kappa para a reprodutibilidade interexaminador do QLF variam entre 0,95 e 0,99, sendo, portanto, esse método facilmente reprodutível e sensível para quantificar lesões cariosas em esmalte limitadas a uma profundidade de 400ųm. Porém, esse aparelho mostra-se limitado para discriminar lesões mais profundas (Fejerskov e Kidd ${ }^{1}$, 2005). Os valores de sensibilidade e especificidade para lesões em esmalte e dentina estão descritos na Tabela II.

Apesar de poucos estudos sobre o QLF, esse método é tido como apropriado para a avaliação dos procedimentos preventivos em pacientes susceptíveis à cárie dentária e muito útil para a monitorização das alterações minerais em lesões incipientes de esmalte (Fejerskov e Kidd ${ }^{1}$, 2005). O 
QLF é o melhor sucedido para a detecção de lesões iniciais de esmalte (Tam e McComb $^{18}, 2001$ ).

\subsection{Fluorescência a laser (DIAGNO- dent $(\mathbb{R})$}

O DIAGNOdent $\AA$ é um dispositivo que se presta ao diagnóstico de cáries oclusais e de superfícies lisas. Com esse aparelho, a superfície do dente é irradiada com um laser vermelho (655 nm), e a emissão de fluorescência da superfície é analisada e quantificada (Figuras 3 e 4). O método é baseado no princípio de que o processo de cárie altera a quantidade de fluorescência dos tecidos dentais que pode ser quantificada (Fejerskov e Kidd' 1 , 2005, George e Stookey ${ }^{17}$, Anttonen et al.22, 2003).

Ao ser incidida na superfície dental, parte da luz laser é absorvida pelo dente e parte é refletida; a intensidade de luz refletida é proporcional à quantidade de mineral existente na estrutura dental; portanto, mudanças na estrutura do dente associadas com a progressão do processo de cárie promovem o aumento na quantia de luz emitida. No DIAGNOdent ${ }^{\circledR}$, essa fluorescência é captada por uma peça de mão, mensurada e então exibida em um visor eletrônico em valores de 0 a 99 (escala de medida), havendo uma correlação direta entre o valor medido e o tamanho de uma lesão de cárie, caso o dente esteja cariado (Fejerskov e Kidd ${ }^{1}$, 2005, Tam e McComb $\left.{ }^{18}, 2001\right)$.

A medição dessa fluorescência causada pela lesão cariosa beneficia o diagnóstico quantitativo da cárie: o valor 0 é característico de uma superfície hígida, enquanto o valor 99 é dado para um dente que está cariado já em dentina (Fejerskov e Kidd $\left.{ }^{1}, 2005\right)$.

A luz fluorescente é medida e sua intensidade indica o tamanho e a profundidade da lesão de cárie (Fejerskov e Kidd ${ }^{1}$, 2005). O valor limiar entre a lesão de cárie limitada ao esmalte e a cárie em dentina fica em torno de 18, sob condições úmidas. O mesmo registro sob condições secas produz valores de corte em torno de 21. Dessa forma, é importante que as condições de hidratação sejam padronizadas para realizações de medidas (Fejerskov e Kidd ${ }^{1}$, 2005). A sensibilidade do DIAGNOdent ${ }^{\circledR}$ com o limiar de 18 a 22 produziu para cáries em dentina de dentes úmidos valores de 0,78 a 0,82 (Tam e McComb $^{18}$, 2001). Esses autores incluem como instruções para o uso do sistema DIAGNOdent ${ }^{\circledR}$ a limpeza da face oclusal a ser examinada, pois placa, tártaro e pigmentações superficiais podem apresentar falsos valores (Tam e McComb ${ }^{18}, 2001$ ). Assim, deve-se fazer a profilaxia com pasta não fluorescente para serem evitadas medidas falso-positivas (Fejerskov e $\mathrm{Kidd}^{1}$, 2005). Mudanças na estrutura física do esmalte, incluindo-se distúrbios de desenvolvimento do dente, produzem, também, leituras incorretas (Tam e McComb ${ }^{18}, 2001$ ).

Estudos mostram que o instrumento exibe reprodutibilidade e sensibilidade excelentes. Entretanto, os resultados dos estudos in vitro indicam também que as leituras podem ser influenciadas por diver-

Tabela I - Comparação entre os valores de kappa para a reprodutibilidade intra e extra-examinador dos métodos de detecção de cárie sengundo os autores pesquisados.

\begin{tabular}{llll}
\hline \hline & Autores & Intra-examinador & Inter-examinador \\
\hline ECM & Kuhnisch et al. ${ }^{14,} 2007$ & 0,69 & 0,62 \\
Tomografia (LCT) & Kalathingal et al.20,2007 & - & 0,69 \\
FOTI & Cortes et al. ${ }^{8}, 2003$ & 0,78 & - \\
Inspeção tátil & Lussi e Francescut ${ }^{7}, 2003$ & $0,24-0,66$ & $(-) 0,08-(-) 0,26$ \\
Bite-wing & Hintze et al. ${ }^{10}, 1998$ & - & $0,48-0,65$ \\
QLF & Fejerskov e Kidd ${ }^{1}, 2005$ & - & $0,95-0,99$ \\
DIAGNOdent & Sheehy et al...23,2001 & 0,97 & - \\
Inspeção visual & Ekstrand et al. $.^{15}, 2005$ & $50-75$ & $32-53$ \\
\hline \hline
\end{tabular}

*FOTI (transiluminação por fibra óptica); ECM (Monitor elétrico de cáries); QLF (Quantitative Light-Induced Fluorescence); OCT (Opti-

cal Coherence Tomograhy); LCT (Tomografia computadorizada local cone beam); TRI (Técnica Radiográfica Interproximal)
LEÃO FILHO JCB

SOUZA TR

MÉTODOS DE

DETECÇÃO DE

CÁRIE: DO

TRADICIONAL

ÀS NOVAS

TECNOLOGIAS DE

EMPREGO CLÍNICO

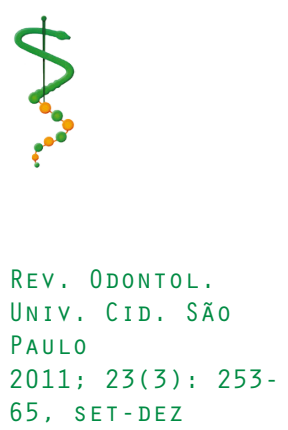


LEÃO FILHO JCB SOUZA TR

MÉTODOS DE DETECÇÃO DE

CÁRIE: DO

TRADICIONAL

ÀS NOVAS

TECNOLOGIAS DE EMPREGO CLINICO sas variáveis, o que pode alterar a reprodutibilidade do aparelho. Essas variáveis incluem o grau de desidratação da lesão e a presença de vários tipos de mancha na superfície dental (George e Stookey ${ }^{17}$ ). Os valores de sensibilidade e especificidade relativos ao DIAGNOdent ${ }^{\circledR}$ estão descritos na Tabela II.

Num estudo de Sheehy et al..$^{23}$ (2001), a reprodutibilidade do método DIAGNOdent ${ }^{\circledR}$, comparada à radiografia conven- cional, foi excelente e a acurácia do diagnóstico foi significativamente melhor que a da radiografia. O DIAGNOdent $\AA$ foi superior ao exame radiográfico na detecção de todos os tipos de lesão de cárie oclusal, tanto em esmalte como em dentina. Porém, quando há cárie na dentina, os dois métodos são adequados (Sheehy et al. ${ }^{23}$, 2001).

O DIAGNOdent $®$ apresentou alto coeficiente de correlação intraexaminado-

Tabela II - Comparação entre os valores de sensibilidade e especificidade dos métodos de detecção de cárie dos autores pesquisados.

\begin{tabular}{|c|c|c|c|c|c|}
\hline \multirow[b]{2}{*}{ Autores } & & \multicolumn{2}{|c|}{ Para Lesões em esmalte } & \multicolumn{2}{|c|}{ Para Lesões em dentina } \\
\hline & & Sensibilidade & Especificidade & Sensibilidade & Especificidade \\
\hline \multirow{6}{*}{$\begin{array}{l}\text { Método } \\
\text { Visual }\end{array}$} & $\begin{array}{l}\text { Cortes et al. }{ }^{8}, \\
2003\end{array}$ & 0,98 & 0,38 & 0,55 & 0,9 \\
\hline & Ferreira Zandona & $0,12-$ & $0,66-$ & - & - \\
\hline & et al. ${ }^{28}, 1998$ & 0,8 & 0,97 & - & - \\
\hline & $\begin{array}{l}\text { Ferreira Zandona } \\
\text { et al. }{ }^{29}, 1998\end{array}$ & 0,03 & 1 & - & - \\
\hline & $\begin{array}{l}\text { Huysmans et al. }{ }^{30} \text {, } \\
1998\end{array}$ & - & - & 0,27 & 1 \\
\hline & $\begin{array}{l}\text { Lussi et al. }{ }^{31}, \\
1999\end{array}$ & - & - & 0,31 & - \\
\hline \multirow{7}{*}{ ECM } & Ferreira Zandona & $0,67-$ & $0,71-$ & - & - \\
\hline & et al. ${ }^{28}, 1998$ & 0,96 & 0,82 & - & - \\
\hline & Lussi et al. ${ }^{31}, 1999$ & 0,87 & 0,64 & 0,92 & 0,78 \\
\hline & $\begin{array}{l}\text { Ashley et al. }{ }^{32}, \\
2000\end{array}$ & - & - & 0,75 & 0,78 \\
\hline & Huysmans et al. ${ }^{30}$, & - & - & $0,58-$ & $0,79-$ \\
\hline & 1998 & - & - & 0,78 & 0,94 \\
\hline & $\begin{array}{l}\text { Fejerskov e Kidd }{ }^{1} \text {, } \\
2005\end{array}$ & - & - & $0,67-0,96$ & $0,71-0,98$ \\
\hline \multirow[t]{3}{*}{ FOTI } & $\begin{array}{l}\text { Cortes et al. }{ }^{8}, \\
2003\end{array}$ & 0,98 & 0,5 & 0,66 & 0,96 \\
\hline & Ferreira Zandona & $0,50-$ & $0,27-$ & - & - \\
\hline & et al. ${ }^{28}, 1998$ & 0,63 & 0,33 & - & - \\
\hline \multirow[t]{4}{*}{ QLF } & $\begin{array}{l}\text { Ferreira Zandona } \\
\text { et al. }{ }^{29}, 1998\end{array}$ & 0,49 & 0,67 & - & - \\
\hline & Hafstrom-Bjork- & - & - & $0,72-$ & $0,79-$ \\
\hline & man et al. ${ }^{33} 1991$ & - & - & 0,76 & 0,81 \\
\hline & Shi et al. ${ }^{34} 2001$ & $0,42-0,46$ & 0,95 & $0,78-0,82$ & 1 \\
\hline \multirow{5}{*}{$\begin{array}{l}\text { DIAG- } \\
\text { NO- } \\
\text { dent }\end{array}$} & Lussi et al. ${ }^{31}$, & $0,83-$ & $0,72-$ & $0,76-$ & $0,79-$ \\
\hline & 1999 & 0,87 & 0,78 & 0,84 & 0,87 \\
\hline & Lussi et al.7, 2003 & 0,96 & - & 0,92 & 0,86 \\
\hline & Sheehy et al. ${ }^{23}$, & $0,42-$ & 0,95 & $0,78-$ & 1 \\
\hline & 2001 & 0,46 & - & 0,82 & - \\
\hline
\end{tabular}

REV, ODONTOL. UNIV, CID, SÃO PAULO $2011 ; 23(3): 253-$ 65, SET-DEZ 
res, 0,97 e 0,96, sob as condições seco e molhado respectivamente, indicando excelente reprodutibilidade, demonstrando ser um método de confiança para o diagnóstico de lesões de cárie (Sheehy et al. ${ }^{23}$, 2001).

Um estudo mostrou que o dispositivo DIAGOdent ${ }^{\circledR}$ não é hábil para diferenciar cáries de hipomineralização (Sheehy et al. ${ }^{23}$, 2001). Portanto, esse aparelho não deve ser utilizado sozinho para detecção de cárie, pois pode induzir a resultados falso-positivos (Fejerskov e Kidd ${ }^{1}, 2005$ ). Além disso, o laser não identifica a atividade da lesão, sendo este mais um motivo para o DIAGNOdent ${ }^{\circledR}$ ser utilizado em conjunto com o exame visual para a correta decisão de tratamento (Anttonen et al.22, 2003).

A fluorescência a laser também pode ser utilizada para monitorar as alterações minerais in vivo em lesões de mancha branca, sendo, portanto, útil para avaliar a eficácia de medidas preventivas em indivíduos susceptíveis à cárie dentária, tais como os pacientes ortodônticos (Fejerskov e Kidd ${ }^{1}$, 2005). O DIAGNOdent ${ }^{\circledR}$ fornece, ainda, um diagnóstico satisfatório em superfícies oclusais com selante, mostrando se há cárie sob o selante. Ele também pode ser usado sem variações satisfatórias tanto em dentes decíduos como em permanentes (Anttonen et al.22, 2003).

Em 2009, numa pesquisa conduzida por Spiguel et al..24 (2009), foram realizados dois estudos para avaliar a utilização do DIAGNOdent ${ }^{\circledR}$ no monitoramento de cáries incipientes: um in vitro e outro in situ. Em ambos, as leituras do DIAGNOdent ${ }^{\circledR}$ foram realizadas e comparadas com medidas de dureza superficial e subsuperficial, antes e após a desmineralização de blocos de esmalte dentário gerada através de um processo de ciclagem de $\mathrm{pH}$. Os valores foram, então, cruzados por um teste de correlação linear, com o objetivo de avaliar se os valores se alteravam de forma coerente, em diferentes graus de mineralização do esmalte. No trabalho in situ, os autores concluíram que a detecção e quantificação de cáries incipientes por fluorescência a laser podem ser utilizadas para a monitorização nos processos de des-remineralização dessas lesões, uma vez que houve correlação estatisticamente significativa entre as leituras de dureza do esmalte e as leituras provenientes do DIAGNOdent ${ }^{\circledR}$. Já no estudo in vitro, essa correlação não existiu.

Mendes e Nicolau ${ }^{25}$ (2004) também avaliaram a capacidade do DIAGNOdent ${ }^{\circledR}$ em monitorar o desenvolvimento de lesões de cárie. Nesse estudo, os valores anteriores e posteriores à desmineralização, realizada pela ciclagem do $\mathrm{pH}$, foram cruzados com a profundidade da lesão observada em microscopia de luz polarizada. Os autores puderam observar, a partir do teste de correlação linear de Pearson, que houve uma correlação estatisticamente significativa entre os valores fornecidos pelo DIAGNOdent ${ }^{\circledR}$ e a profundidade das lesões produzidas. Portanto, o aparelho se mostrou eficiente na detecção de lesões incipientes, podendo ser útil no monitoramento dessas lesões, pois tal método é capaz de quantificar a lesão de acordo com sua profundidade.

\section{Técnica radiográfica interproximal (Bi-} te-wing)

Atualmente, a técnica radiográfica interproximal (TRI) tem sido utilizada como um método de detecção de cáries auxiliar à inspeção visual, de forma muito comum e difundida na prática clínica. Na imagem radiográfica, a perda de estrutura dentária (seja causada pela cárie ou por qualquer outro agente) é percebida pela existência de radioluscências. Além da detecção de cáries, a TRI também possibilita a visualização de excessos nas restaurações e a avaliação das cristas ósseas alveolares (Fejerskov e Kidd ${ }^{1}$, 2005, Marcucci ${ }^{26}$, 2005).

Apesar de ser uma técnica muito difundida, a TRI não deve ser utilizada de forma exclusiva e individual para a detecção de cáries, pois quando é necessário tomar a decisão pelo uso de um tratamento invasivo ou por um não invasivo, a aparência clínica visual da lesão deve ter maior peso sobre a decisão do rumo do tratamento (Hintze et al. ${ }^{10}$, 1998).

Quando numa radiografia a radioluscência é vista na metade mais profunda da dentina, a probabilidade de haver clinicamente cavitação sob a inspeção visual chega a $100 \%$. Por outro lado, quando a
LEÃO FILHO JCB

SOUZA TR

MÉTODOS DE

DETECÇÃO DE

CÁRIE: DO

TRADICIONAL

ÀS NOVAS

TECNOLOGIAS DE

EMPREGO CLÍNICO
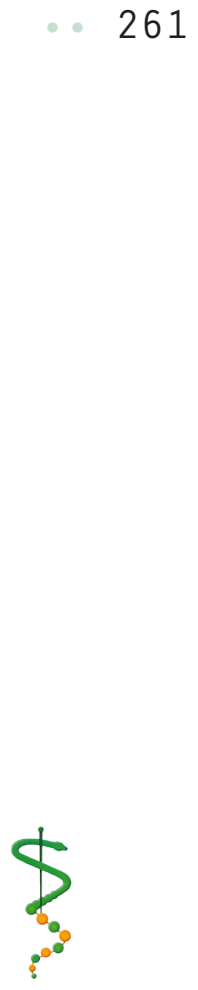

REV, ODONTOL.

UNIV, CID. SÃO

PAULO

2011; 23(3): 253-

65, SET-DEZ 
LEÃO FILHO JCB

SOUZA TR

MÉTODOS DE

DETECÇÃO DE

CÁRIE: DO

TRADICIONAL

ÀS NOVAS

TECNOLOGIAS DE

EMPREGO CLÍNICO

$262 \ldots$

REV. ODONTOL,

UNIV. CID. SÃO

PAULO

$2011 ; 23(3): 253-$

65, SET-DEZ radiolucidez é observada na metade mais superficial da dentina, a chance de haver clinicamente uma cavitação varia entre 41 e $79 \%$. De uma forma geral, esses valores demonstram que existe maior chance de lesões cavitadas quando a radiolucidez atinge a dentina; porém a presença dessa radiolucidez na metade mais superficial da dentina não constitui fator conclusivo para a presença de cavitações (Hintze et al. $\left.{ }^{10}, 1998\right)$.

Esse método possui relativamente uma alta sensibilidade. Na Tabela II aparecem os valores de especificidade e sensibilidade para a identificação de lesões cavitadas pela TRI. Os valores de kappa para a concordância interobservadores variam entre 0,48 e 0,65 para a TRI (Hintze et al. ${ }^{10}$, 1998).

A incessante busca por inovações tecnológicas levou ao desenvolvimento das chamadas radiografias digitais. Nessa nova técnica, o filme radiográfico é substituído por sensores que levam os dados captados para um computador, e lá são transformados em imagens digitais exibidas em um monitor (Freitas ${ }^{27}$, 2000).

Essas imagens digitais, entretanto, possuem menor resolução espacial que aquela observada nos filmes radiográficos tradicionais, pois o espectro de tons de cinza, para as imagens digitais, é limitado a 256 diferentes tons; já no filme radiográfico convencional o espectro de tons de cinza ultrapassa o valor de um milhão de tons. Por esse motivo, o desempenho dos filmes convencionais ultrapassa o das imagens digitais, que produzem um maior número de diagnósticos incorretos (Fejerskov e Kidd $\left.{ }^{1}, 2005\right)$.

As radiografias digitais possuem a vantagem de facilitar a manipulação das imagens, além de proporcionar a execução da técnica radiográfica digital por subtração. Esta última baseia-se na subtração de tons de cinza de uma imagem radiográfica digital (produzida num segundo momento) de outra imagem (produzida num primeiro momento). Se o resultado da subtração for zero, significa que não houve modificações na estrutura dental, porém a existência de um resultado diferente de zero é indicativo de mudanças na constituição do tecido dental. Esse método é, portanto,
Tabela III - Comparação entre os valores de sensibilidade e especificidade do LCT e da radiografia Bite-wing.

\begin{tabular}{ccc}
\hline \hline & Sensibilidade & Especificidade \\
\hline LCT & 0,7 & 0,82 \\
Bite-wing & 0,51 & 0,92 \\
\hline \hline
\end{tabular}

uma poderosa ferramenta para o diagnóstico de cáries primárias e secundárias (Fejerskov e Kidd ${ }^{1}$, 2005).

Esse método por subtração pode ser muito útil na proservação de lesões, quando o sucesso do procedimento executado depende de uma monitorização eficaz dessas lesões. Essas situações podem ser exemplificadas quando as lesões forem tratadas preventivamente ou quando existe uma lesão cariosa selada abaixo de uma restauração (Ricketts et al. ${ }^{21}, 2007$ ).

Um estudo recente afirma que a eficácia da técnica radiográfica digital por subtração é superior à técnica de comparação entre duas radiografias emparelhadas lado a lado, para a determinação da progressão de desmineralização. A explicação para esse fato é que a comparação de imagens emparelhadas é subjetiva (Ricketts et al. ${ }^{21}$, 2007). Apesar das vantagens do método, ele ainda não é utilizado rotineiramente nas clínicas odontológicas devido, principalmente, à dificuldade de se encontrar o alinhamento exato entre as imagens obtidas (Fejerskov e Kidd $\left.{ }^{1}, 2005\right)$.

6. Corantes como método de detecção de cárie

Os corantes utilizados na dentística clínica são capazes de aumentar o contraste entre tecidos normais e alterados pela cárie, aumentando, assim, a precisão no diagnóstico de lesões cariosas. Porém, mesmo sendo este um método eficaz na distinção de tecido sadio e tecido alterado, ele possui muitas desvantagens, tais como reações de toxicidade e criação de manchas (irreversíveis ou difíceis de serem removidas) nos tecidos dentais (Marinho e Pereira ${ }^{6}$, 1998).

Atualmente, o uso de corantes como método de detecção de cárie não é muito indicado, visto que os corantes não diferenciam a dentina infectada da desmineralizada, fazendo com que haja remoção 
desnecessária de tecido dental sadio. Além disso, em casos de lesões muito profundas, o corante possui alta afinidade pela dentina circumpulpar, o que aumenta o risco de exposição pulpar pelo desgaste do tecido evidenciado, que não se constitui necessariamente de tecido infectado (Mialhe et al. $\left.{ }^{2}, 2005\right)$.

\section{CONCLUSÃO}

O desenvolvimento de métodos para a detecção de lesões incipientes de cárie, que sejam reprodutíveis (intra e interexaminadores) e acurados para o diagnóstico e acompanhamento da progressão dessas lesões, é crítico para suprir as necessidades clínicas, devido ao surgimento de um novo perfil de cárie.

Atualmente, tem-se dado mais ênfase para a aparência clínica da lesão, quando é necessário tomar uma decisão sobre a forma de tratamento a ser aplicada (se invasivo ou não). Isso porque, além de identificar as lesões, o método de inspeção visual é capaz de determinar a atividade da lesão em questão.
Já o método de inspeção tátil (com o uso do explorador) é considerado antiquado e deve ter o seu uso desencorajado. Esse método apresenta desvantagens que incluem sua baixa sensibilidade, ou seja, pobre capacidade de identificar lesões cariosas, além de tornar possível a quebra da integridade da superfície do esmalte pela sondagem com o explorador.

Outros métodos podem ser utilizados como auxiliares à inspeção visual. Muitas das tecnologias mais recentes desenvolvidas para a detecção de cárie não estão disponíveis no mercado, porque apresentam, em geral, um custo elevado, além de possuírem um protocolo mais trabalhoso, necessitando de maior tempo clínico para serem utilizadas do que os métodos convencionais.

A comparação entre os novos métodos pesquisados pode ser feita observando-se os valores de kappa para a reprodutibilidade intra e interexaminadores, a especificidade e a sensibilidade, descritos nas Tabelas I e II.

\section{REFERÊNCIAS}

1. Fejerskov O, Kidd E. Cárie dentária: a doença e seu tratamento clínico. São Paulo: Santos; 2005.

2. Mialhe FL, Bosquiroli V, Silva JO. Conhecimento e utilização de métodos de detecção de lesões cariosas por cirurgiões-dentistas. Varia Scientia 2005 5(10):23-33.

3. Ismail Al. Clinical diagnosis of precavitated carious lesions. Community Dent Oral Epidemiol 1997 Feb;25(1):13-23.

4. Nyvad B. Diagnosis versus detection of caries. Caries Res 2004 May-Jun;38(3):192-8.

5. Murdoch-Kinch CA, McLean ME. Minimally invasive dentistry. J Am Dent Assoc 2003 Jan;134(1):87-95.

6. Marinho V, Pereira G. Cárie: diagnóstico e plano de tratamento. R Un Alfenas 1998 4):27-37.

7. Lussi A, Francescut P. Performance of conventional and new methods for the detection of occlusal caries in deciduous teeth. Caries Res 2003 Jan-Feb;37(1):2-7.

8. Cortes DF, Ellwood RP, Ekstrand KR. An in vitro comparison of a combined FOTI/ visual examination of occlusal caries with other caries diagnostic methods and the effect of stain on their diagnostic performance. Caries Res 2003 Jan-Feb;37(1):8-16.

9. Kidd EA, Fejerskov O. What constitutes dental caries? Histopathology of carious enamel and dentin related to the action of cariogenic biofilms. J Dent Res 200483 Spec No C(C35-8.

LEÃO FILHO JCB

SOUZA TR

MÉTODOS DE

DETECÇÃO DE

CÁRIE: DO

TRADICIONAL

ÀS NOVAS

teCNOLOGIAS DE

EMPREGO CLÍNICO

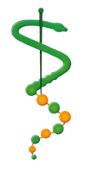

REV. ODONTOL.

UNIV. CID. SÃo

PAULO

2011; 23(3): 253 -

65, SET-DEZ 
LEÃO FILHO JCB SOUZA TR

MÉTODOS DE

DETECÇÃO DE

CÁRIE: DO

TRADICIONAL

ÀS NOVAS

TECNOLOGIAS DE

EMPREGO CLINICO

264

I SSN $1983-5183$

10. Hintze H, Wenzel A, Danielsen B, Nyvad B. Reliability of visual examination, fibre-optic transillumination, and bite-wing radiography, and reproducibility of direct visual examination following tooth separation for the identification of cavitated carious lesions in contacting approximal surfaces. Caries Res 1998 32(3):204-9.

11. Eggertsson H, Analoui M, van der Veen M, Gonzalez-Cabezas C, Eckert G, Stookey G. Detection of early interproximal caries in vitro using laser fluorescence, dye-enhanced laser fluorescence and direct visual examination. Caries Res 1999 May-Jun;33(3):227-33.

12. De Araujo FB, Rosito DB, Toigo E, dos Santos CK. Diagnosis of approximal caries: radiographic versus clinical examination using tooth separation. Am J Dent 1992 Oct;5(5):245-8.

13. Haak R, Wicht MJ, Hellmich M, Gossmann A, Noack MJ. The validity of proximal caries detection using magnifying visual aids. Caries Res 2002 Jul-Aug;36(4):249-55.

14. Kuhnisch J, Dietz W, Stosser L, Hickel R, Heinrich-Weltzien R. Effects of dental probing on occlusal surfaces--a scanning electron microscopy evaluation. Caries Res 2007 41(1):43-8.

15. Ekstrand KR, Ricketts DN, Longbottom C, Pitts NB. Visual and tactile assessment of arrested initial enamel carious lesions: an in vivo pilot study. Caries Res 2005 May-Jun;39(3):173-7.

16. Kuhnisch J, Heinrich-Weltzien R, Tabatabaie M, Stosser L, Huysmans MC. An in vitro comparison between two methods of electrical resistance measurement for occlusal caries detection. Caries Res 2006 40(2):104-11.

17. George K, Stookey M. The evolution of caries detection. [cited 201018 ago]; Available from: http://www.dimensionsofdentalhygiene.com/ddhright.aspx?id=122

18. Tam LE, McComb D. Diagnosis of occlusal caries: Part II. Recent diagnostic technologies. J Can Dent Assoc 2001 Sep;67(8):459-63.

19. Otis LL, Everett MJ, Sathyam US, Colston BW, Jr. Optical coherence tomography: a new imaging technology for dentistry. J Am Dent Assoc 2000 Apr;131(4):511-4.

20. Kalathingal SM, Mol A, Tyndall DA, Caplan DJ. In vitro assessment of cone beam local computed tomography for proximal caries detection. Oral Surg Oral Med Oral Pathol Oral Radiol Endod 2007 Nov;104(5):699-704.

21. Ricketts DN, Ekstrand KR, Martignon S, Ellwood R, Alatsaris M, Nugent Z. Accuracy and reproducibility of conventional radiographic assessment and subtraction radiography in detecting demineralization in occlusal surfaces. Caries Res 2007 41(2):121-8.

22. Anttonen V, Seppa L, Hausen H. Clinical study of the use of the laser fluorescence device DIAGNOdent for detection of occlusal caries in children. Caries Res 2003 Jan-Feb;37(1):17-23.

23. Sheehy EC, Brailsford SR, Kidd EA, Beighton D, Zoitopoulos L. Comparison between visual examination and a laser fluorescence system for in vivo diagnosis of occlusal caries. Caries Res 2001 Nov-Dec;35(6):421-6.

24. Spiguel MH, Tovo MF, Kramer PF, Franco KS, Alves KM, Delbem AC. Evaluation of laser fluorescence in the monitoring of the initial stage of the de-/remineralization process: an in vitro and in situ study. Caries Res 2009 43(4):302-7.

REV. ODONTOL.

UNIV. CID. SÃO PAULO

25. Mendes FM, Nicolau J. Utilization of laser fluorescence to monitor caries lesions development in primary teeth. J Dent Child (Chic) 2004 May-Aug;71(2):139-42. 
26. Marcucci G. Fundamentos de odontologia: estomatogia. Rio de Janeiro: Guanabara Koogan; 2005.

27. Freitas L. Radiologia bucal: técnicas e interpretação. 2 ed. São Paulo: Pancast; 2000.

28. Ferreira Zandona AG, Analoui M, Schemehorn BR, Eckert GJ, Stookey GK. Laser fluorescence detection of demineralization in artificial occlusal fissures. Caries Res 1998 32(1):31-40.

29. Ferreira Zandona AG, Analoui M, Beiswanger BB, Isaacs RL, Kafrawy AH, Eckert G), et al. An in vitro comparison between laser fluorescence and visual examination for detection of demineralization in occlusal pits and fissures. Caries Res 1998 32(3):210-8.

30. Huysmans MC, Longbottom C, Pitts N. Electrical methods in occlusal caries diagnosis: An in vitro comparison with visual inspection and bite-wing radiography. Caries Res 1998 32(5):324-9.

31. Lussi A, Imwinkelried S, Pitts N, Longbottom C, Reich E. Performance and reproducibility of a laser fluorescence system for detection of occlusal caries in vitro. Caries Res 1999 Jul-Aug;33(4):261-6.

32. Ashley PF, Ellwood RP, Worthington HV, Davies RM. Predicting occlusal caries using the Electronic Caries Monitor. Caries Res 2000 Mar-Apr;34(2):201-3.

33. Hafstrom-Bjorkman U, Sundstrom F, Angmar-Mansson B. Initial caries diagnosis in rat molars, using laser fluorescence. Acta Odontol Scand 1991 Feb;49(1):27-33.

34. Shi XQ, Tranaeus S, Angmar-Mansson B. Validation of DIAGNOdent for quantification of smooth-surface caries: an in vitro study. Acta Odontol Scand 2001 Apr;59(2):74-8.

Recebido em: 09/09/2010

Aceito em: 05/04/2011

LEÃO FILHO JCB

SOUza tR

MÉTODOS DE

DETECÇÃO DE

CÁRIE: DO

TRADICIONAL

ÀS NOVAS

teCNOLOGIAS DE

EMPREGO CLÍNICO

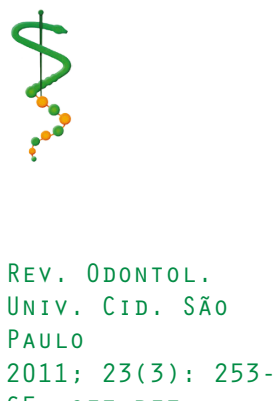

65 , SET-DEZ 